\title{
ON CONVOLUTIONS IN HILBERT SPACES
}

\author{
B. KANGUZHIN, M. RUZHANSKY, AND N. TOKMAGAMBETOV
}

0.1. Introduction. Let $\mathcal{H}$ be a separable Hilbert space. Denote by $\mathcal{U}:=\left\{u_{\xi} \mid u_{\xi} \in\right.$ $\mathcal{H}\}_{\xi \in \mathcal{I}}$ and $\mathcal{V}:=\left\{v_{\xi} \mid v_{\xi} \in \mathcal{H}\right\}_{\xi \in \mathcal{I}}$ collections of elements of $\mathcal{H}$ parametrised by a discrete set $\mathcal{I}$. We assume that the system $\mathcal{U}$ is a basis of the space $\mathcal{H}$ and the system $\mathcal{V}$ is biorthogonal to $\mathcal{U}$ in $\mathcal{H}$. Then $\left(u_{\xi}, v_{\eta}\right)_{\mathcal{H}}=\delta_{\xi \eta}$, where $\delta_{\xi \eta}$ is the Kronecker delta, equal to 1 for $\xi=\eta$, and to 0 otherwise. In this case from the classical Bari's work [3] ((see also Gelfand's paper [7]) it follows that the system $\mathcal{V}$ is also basis in $\mathcal{H}$.

We define $\mathcal{U}$ - and $\mathcal{V}$-convolutions in the following form:

$$
f \star \mathcal{U} g:=\sum_{\xi \in \mathcal{I}}\left(f, v_{\xi}\right)\left(g, v_{\xi}\right) u_{\xi}
$$

and

$$
h \star \mathcal{V} j:=\sum_{\xi \in \mathcal{I}}\left(h, u_{\xi}\right)\left(j, u_{\xi}\right) v_{\xi}
$$

for appropriate elements $f, g, h, j \in \mathcal{H}$.

0.2. Example. We give an example, which can be considered as an extension of the periodic calculus studied in [12].

Consider an operator $\mathrm{O}_{h}^{(1)}$ defined by the action $\mathrm{O}_{h}^{(1)}:=-i \frac{d}{d x}$ for $h>0$, on the interval $(0,1)$ with the boundary condition $h y(0)=y(1)$. When $h=1, \mathrm{O}_{1}^{(1)}$ is defined by the periodic condition, and elements of systems $\mathcal{U}$ and $\mathcal{V}$ are eigenfunctions of the operator $\mathrm{O}_{1}^{(1)}$ and its conjugate $\mathrm{O}_{1}^{(1)^{*}}$ coincide, and are given by $\mathcal{U}=\mathcal{V}=$ $\left\{u_{j}(x)=e^{2 \pi i x j}, j \in \mathbb{Z}\right\}$. That is, we get the classical Fourier analysis on a closed circle. Corresponding pseudo-differential calculus was developed in [12], which based on the previous investigations of Agranovich [1,2] and others.

For $h \neq 1$ the operator $\mathrm{O}_{h}^{(1)}$ is not self-adjoint. The spectral properties of $\mathrm{O}_{h}^{(1)}$ are well-known (see Titchmarsh [16] and Cartwright [4]). The corresponding biorthogonal families of eigenfunctions of $\mathrm{O}_{h}^{(1)}$ and its adjoint are given by $\mathcal{U}=\left\{u_{j}(x)=\right.$ $\left.h^{x} e^{2 \pi i x j}, j \in \mathbb{Z}\right\}$ and $\mathcal{V}=\left\{v_{j}(x)=h^{-x} e^{2 \pi i x j}, j \in \mathbb{Z}\right\}$, respectively. They form Riesz bases in $\mathcal{H}=L^{2}(0,1)$.

In this case, the convolution (0.1) has the form

$$
\left(f \star_{\mathcal{U}} g\right)(x)=\int_{0}^{x} f(x-t) g(t) d t+\frac{1}{h} \int_{x}^{1} f(1+x-t) g(t) d t,
$$

which coincides with the standard convolution when $h=1$. This convolution was investigated in papers $[9,11]$. 
0.3. Formulation of results. We are aiming at discussion of convolutions from more abstract point of view, that is, when there is only given a Riesz basis in the Hilbert space without any additional assumptions on the operator with eigenfunctions form this basis.

Easy to check, that the expressions $(0.1)$ and 0.2 are correctly defined:

Theorem 0.1. Let $f \star_{\mathcal{U}} g$ and $h \star_{\mathcal{V}} j$ are given by formulae (0.1) and (0.2), respectively. Suppose that the families of functions $\mathcal{U}$ and $\mathcal{V}$ are uniformly bounded in $\mathcal{H}$. Then there exists a constant $M>0$ such that the inequalities hold:

$$
\left\|f \star_{\mathcal{U}} g\right\|_{\mathcal{H}} \leq M \sup _{\xi \in \mathcal{I}}\left\|u_{\xi}\right\|_{\mathcal{H}}\|f\|_{\mathcal{H}}\|g\|_{\mathcal{H}}, \quad\left\|h \star_{\mathcal{V}} j\right\|_{\mathcal{H}} \leq M \sup _{\xi \in \mathcal{I}}\left\|v_{\xi}\right\|_{\mathcal{H}}\|h\|_{\mathcal{H}}\|j\|_{\mathcal{H}},
$$

for all $f, g, h, j \in \mathcal{H}$.

Let us introduce $\mathcal{U}$ - and $\mathcal{V}$-Fourier transforms by formulae

$$
\mathcal{F}_{\mathcal{U}}(f)(\xi):=\left(f, v_{\xi}\right)=: \widehat{f}(\xi)
$$

and

$$
\mathcal{F}_{\mathcal{V}}(g)(\xi):=\left(g, u_{\xi}\right)=: \widehat{g}_{*}(\xi),
$$

respectively, for any $f, g \in \mathcal{H}$ and for arbitrary $\xi \in \mathcal{I}$. The inverse transforms have the following forms

$$
\left(\mathcal{F}_{\mathcal{U}}^{-1} a\right)(x):=\sum_{\xi \in \mathcal{I}} a(\xi) u_{\xi}
$$

and

$$
\left(\mathcal{F}_{\mathcal{V}}^{-1} a\right)(x):=\sum_{\xi \in \mathcal{I}} a(\xi) v_{\xi}
$$

Now we formulate the statement about the relationship between $\mathcal{U}$-and $\mathcal{V}$-convolutions, and Fourier transforms:

Theorem 0.2. For all $f, g, h, j \in \mathcal{H}$ we have

$$
\widehat{f \star \mathcal{U} g}=\widehat{f} \widehat{g}, \quad \widehat{h \star \mathcal{V}} j_{*}=\widehat{h}_{*} \widehat{j}_{*} .
$$

Thus, the convolutions are commutative and associative.

Let $K: \mathcal{H} \times \mathcal{H} \rightarrow \mathcal{H}$ be a bilinear operation. If for all $f, g \in \mathcal{H}$ the form $K(f, g)$ satisfies

$$
\widehat{K(f, g)}=\widehat{f} \widehat{g}
$$

then $K$ is a $\mathcal{U}$-convolution, that is $K(f, g)=f *_{\mathcal{U}} g$.

Analogously, if $K(f, g)$ satisfies the property

$$
\widehat{K(f, g)})_{*} \widehat{f}_{*} \widehat{g}_{*}
$$

then $K$ is a $\mathcal{V}$-convolution, that is $K(f, g)=f * \mathcal{V} g$.

In particular, the last part of Theorem means that the convolution $(0.1)$ is uniquely determined by the condition: the Fourier transform of the convolution of elements is a product of Fourier transforms of elements. 
0.4. Discussion. Let us discuss some additional aspects of the arisen convolutions.

1. By putting $\mathcal{H}=L^{2}(X)$ for some set $X$, we write

$$
\left(f \star_{\mathcal{U}} g\right)(x)=\int_{X} \int_{X} F(x, y, z) f(y) g(z) d y d z,
$$

where

$$
F(x, y, z)=\sum_{\xi \in \mathcal{I}} u_{\xi}(x) \overline{v_{\xi}(y)} \overline{v_{\xi}(z)} .
$$

Here, we understand the integral $(0.10)$ and the last series in the generalised function sense. In the example from Section 0.2 , when $h=1$, we have $F(x, y, z)=\delta(x-y-z)$ (see [12]).

3. Obviously, convolutions $(0.1)$ and $(0.2)$ are commutative and associative. Moreover, they possess many properties of the standard convolution. The most important one is mapping to a product of Fourier transforms associated with $\mathcal{U}$ and $\mathcal{V}$.

4. Very often biorthogonal families arise as systems of eigenfunctions densely defined non-self-adjoint operators in $\mathcal{H}$, and the corresponding notion of the convolution leads to the development of the associated Fourier Analysis. In this case, when eigenfunctions do not have zeros, then the corresponding global analysis of pseudodifferential operators is developed in the recent paper [13]. Without zero condition is removed in [14], and an application of the analysis to the wave equation for Landau Hamiltonian is given in [15]. Also, we note papers [5, 6], where properties of pseudodifferential operators on manifolds with and without boundary are studied.

5. We also consider a convolution generated by Ionkin operator from [8]. Ionkin operator $\mathcal{Y}$ is defined in $\mathcal{H}:=L^{2}(0,1)$, and generated by the differential expression $-\frac{d^{2}}{d x^{2}}, x \in(0,1)$, with boundary conditions $u(0)=0, u^{\prime}(0)=u^{\prime}(1)$. The operator has the system of eigen- and associated function

$$
u_{0}(x)=x, \quad u_{2 \xi-1}(x)=\sin (2 \pi \xi x), \quad u_{2 \xi}(x)=x \cos (2 \pi \xi x), \xi \in \mathbb{N},
$$

which forms a basis in $L^{2}(0,1)$. Denote them by $\mathcal{U}$. The corresponding biorthogonal basis can be given as

$$
v_{0}(x)=2, \quad v_{2 \xi-1}(x)=4(1-x) \sin (2 \pi \xi x), \quad v_{2 \xi}(x)=4 \cos (2 \pi \xi x), \quad \xi \in \mathbb{N},
$$

for more information we refer to [8]. We define $\mathcal{Y}$-convolution by the formula

$$
\begin{aligned}
f \star \mathcal{Y} g(x): & =\frac{1}{2} \int_{x}^{1} f(1+x-t) g(t) d t \\
+ & \frac{1}{2} \int_{1-x}^{1} f(x-1+t) g(t) d t+\int_{0}^{x} f(x-t) g(t) d t \\
& \quad-\frac{1}{2} \int_{0}^{1-x} f(1-x-t) g(t) d t+\frac{1}{2} \int_{0}^{x} f(1+t-x) g(t) d t .
\end{aligned}
$$

Similar $\mathcal{Y}$-convolution is studied in the paper [10]. In particular, it satisfies the following properties:

$$
\mathcal{Y}(f \star \mathcal{Y} g)=(\mathcal{Y} f) \star \mathcal{Y} g=f \star \mathcal{Y}(\mathcal{Y} g)
$$

For the basis

$$
\mathcal{U}:=\left\{u_{\xi}: u_{0}(x)=x, u_{2 \xi-1}(x)=\sin (2 \pi \xi x), u_{2 \xi}(x)=x \cos (2 \pi \xi x), \xi \in \mathbb{N}\right\},
$$


we have $\widehat{f \star y} g(0)=\widehat{f}(0) \widehat{g}(0), \widehat{f \star y g}(2 \xi)=\widehat{f}(2 \xi) \widehat{g}(2 \xi)$, and $\widehat{f \star y} g(2 \xi-1)=\widehat{f}(2 \xi-$ $1) \widehat{g}(2 \xi)+\widehat{f}(2 \xi) \widehat{g}(2 \xi)+\widehat{f}(2 \xi) \widehat{g}(2 \xi-1), \xi \in \mathbb{N}$. Thus, by Theorem 0.2 , it follows that the $\mathcal{Y}$-convolution coincides with the $\mathcal{U}$-convolution.

\section{References}

1. M. S. Agranovich, Funkts. analiz i ego pril., 13:4 (1979), 54-56. 2. M. S. Agranovich, Trudy MMO, 47 (1984), 22-67. 3. N. K. Bari, Uch. zapiski Mosk. gos. unta. Matematika, 148:4 (1951), 69-107. 4. M. L. Cartwright. Quart. J. Math., Oxford Ser., 1(1):38-59, 1930. 5. J. Delgado, M. Ruzhansky. J. Anal. Math. (https://arxiv.org/abs/1404.6479) 6. J. Delgado, M. Ruzhansky and N. Tokmagambetov. J. Math. Pures Appl., 107:6 (2017), 758-783. 7. I. M. Gelfand, Uch. zapiski Mosk. gos. un-ta. Matematika, 148:4 (1951), 224-225. 8. N. I. Ionkin, Differents. uravneniya, 13:2 (1977), 294-304. 9. B. Kanguzhin and N. Tokmagambetov. Fourier Analysis: Trends in Mathematics, pages 235-251. Birkhäuser Basel AG, Basel, 2014. 10. B. Kanguzhin and N. Tokmagambetov. Ufimsk. Mat. Zh., 7(4):80-92. 2015. 11. B. Kanguzhin, N. Tokmagambetov, and K. Tulenov. Complex Var. Elliptic Equ., 60(1):107-117, 2015. 12. M. Ruzhansky and V. Turunen. J. Fourier Anal. Appl., 16(6):943-982, 2010. 13. M. Ruzhansky and N. Tokmagambetov. Int. Math. Res. Not. IMRN, 2016(12):3548-3615, 2016. 14. M. Ruzhansky and N. Tokmagambetov. Math. Model. Nat. Phenom., 12(1):115-140, 2017. 15. M. Ruzhansky and N. Tokmagambetov. Lett. Math. Phys., 107:4 (2017), 591-618. 16. E. C. Titchmarsh. Proc. London Math. Soc., 25:283-302, 1926.

BALTABEK KanguZHin:

aL-FARABi Kazakh National University

71 al-Farabi aVe., Almaty, 050040

KAZAKHSTAN

E-mail address kanbalta@mail.ru

Michael Ruzhansky:

Department of Mathematics

IMPERIAL COLLEGE LONDON

180 Queen's Gate, London, SW7 2AZ

United Kingdom

E-mail address m.ruzhansky@imperial.ac.uk

Niyaz Tokmagambetov:

aL-Farabi Kazakh National University

71 al-Farabi aVe., Almaty, 050040

KAZAKHSTAN,

AND

Department of Mathematics

IMPERIAL COLLEGE LONDON

180 QueEn's Gate, London, SW7 2AZ

UNITED KINGDOM

E-mail address n.tokmagambetov@imperial.ac.uk 